\title{
Communication Estimation for Hardware/Software Codesign
}

\author{
Knudsen, Peter Voigt; Madsen, Jan
}

Published in:

Hardware/Software Codesign, 1998. (CODES/CASHE '98) Proceedings of the Sixth International Workshop on

Link to article, DOI:

10.1109/HSC.1998.666238

Publication date:

1998

Document Version

Publisher's PDF, also known as Version of record

Link back to DTU Orbit

Citation (APA):

Knudsen, P. V., \& Madsen, J. (1998). Communication Estimation for Hardware/Software Codesign. In Hardware/Software Codesign, 1998. (CODES/CASHE '98) Proceedings of the Sixth International Workshop on (pp. 55-59). IEEE Computer Society Press. https://doi.org/10.1109/HSC.1998.666238

\section{General rights}

Copyright and moral rights for the publications made accessible in the public portal are retained by the authors and/or other copyright owners and it is a condition of accessing publications that users recognise and abide by the legal requirements associated with these rights.

- Users may download and print one copy of any publication from the public portal for the purpose of private study or research.

- You may not further distribute the material or use it for any profit-making activity or commercial gain

- You may freely distribute the URL identifying the publication in the public portal 


\title{
Communication Estimation for Hardware/Software Codesign
}

\author{
Peter Voigt Knudsen and Jan Madsen \\ Department of Information Technology, Technical University of Denmark \\ pvk@it.dtu.dk,jan@it.dtu.dk
}

\begin{abstract}
This paper presents a general high level estimation model of communication throughput for the implementation of a given communication protocol. The model, which is part of a larger model that includes component price, software driver object code size and hardware driver area, is intended to be general enough to be able to capture the characteristics of a wide range of communication protocols and yet to be sufficiently detailed as to allow the designer or design tool to efficiently explore tradeoffs between throughput, bus widths, burst/non-burst transfers and data packing strategies. Thus it provides a basis for decision making with respect to communication protocols/components and communication driver design in the initial design space exploration phase of a co-synthesis process where a large number of possibilities must be examined and where fast estimators are therefore necessary. The full model allows for additional (money)cost, software code size and hardware area tradeoffs to be examined.
\end{abstract}

\section{Introduction}

This paper presents the underlying estimation model for a communication estimation tool which extends the current communication estimation capabilities of the LYCOS [2] co-synthesis system. The model is the basis of a high level communication library that for each supported processing unit and for each supported protocol captures performance/area/price and other characteristics of the necessary drivers and of the communication channel. Our aim is to utilize this library in a communication estimation tool that will work together with the other estimation/partitioning tools in LYCOS as part of the design space exploration/cosynthesis cycle. Most current approaches to co-synthesis consider communication synthesis to be a final step in the co-synthesis trajectory [1][3][4]. For instance, [1] presents communication synthesis as an allocation problem to be solved after system-level partitioning whereas we integrate communication synthesis with design space exploration and system level partitioning. For example, we wish to be able to trade off a fast and expensive communication protocol for a slow but cheaper protocol and a faster co-processor, if that is feasible. This should not be done after system level partitioning as the level of communication overhead between system components influences what the best partition is. For this we need fast estimators of the kind presented in this paper. [6] models communication at various levels of abstraction which enables multi-level system simulation to verify correct behavior given the selected communication components/protocols, but the question of how to select the best combination of communication components/protocols still needs to be addressed. Our communication model in combination with the estimation tool helps the designer/design tool answer this question.

\section{The communication model}

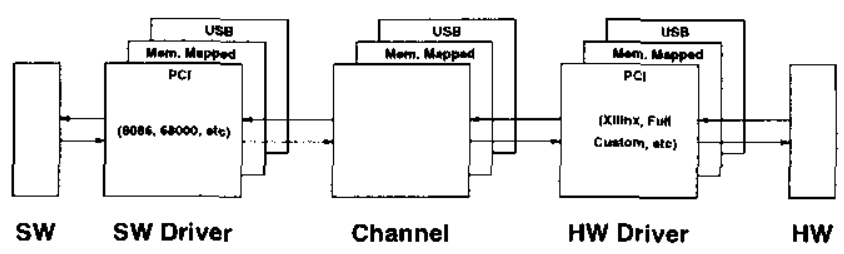

Figure 1. Communication model overview.

Figure 1 shows our model of point to point communication. The figure shows communication in a processor/coprocessor target architecture, but the model is not limited to this architecture - it can be used to model and estimate communication overhead in any architecture where a connection between two processing elements has been established. The time overhead of establishing such a connection (arbitration, etc.) is currently not modeled/estimated. Note that, in contrast to prior work, we consider the possible performance degradation imposed by the hardware/software drivers, and not only the characteristics of the channel.

For simplicity, we consider communication in one direction only in this paper. In general, some of the model parameters will depend on the transmission direction. For instance, a PCI bus master read is slower than a write, so the parameters that model channel transmission delay exist 
in both a "read" version and a "write" version in the full model.

\subsection{Driver transmission delay model}

Figure 2 defines the parameters which are used to estimate driver transmission delay. The driver receives $n_{t}$ input

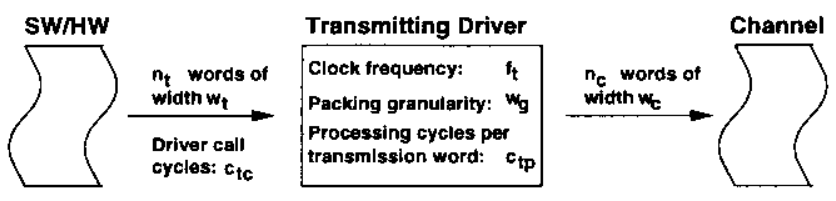

Figure 2. Driver transmission parameters.

words for transmission and produces $n_{c}$ channel words. In order to do so, it may have to pack or split driver input words in order to fit the channel bit width $w_{c}$ and it may have to perform other kinds of data processing. The packing granularity $w_{g}$ influences the transmission processing delay and is defined in section 2.6. Given the clock frequency of the transmitting processor, $f_{t}$, the number of cycles, $c_{t c}$, it requires to call the driver for transmission (transfer arguments to, transfer execution flow to, etc.) and the number of transmission processing (packing/splitting/etc.) cycles per driver input word, $c_{t}$, we can write the total driver transmission delay as

$$
t_{t d}=\left(c_{t c}+c_{t p} n_{t}\right) / f_{t}
$$

\subsection{Channel transmission delay model}

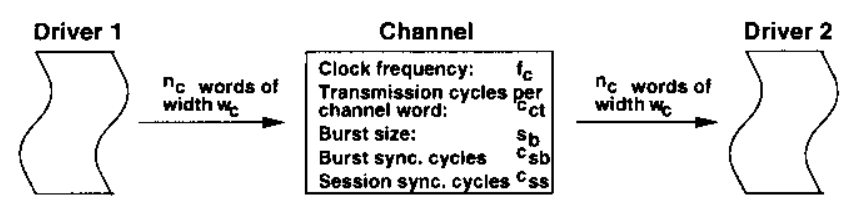

Figure 3. Channel transmission parameters.

Assume that the number of transmitted channel words $n_{c}$ and the number of required synchronization cycles $c_{c s}$ are known (formulas for these will be derived in sections 2.5 and 2.6). Given the clock frequency of the channel $f_{c}$ and the number of transmission cycles per channel word $c_{c t}$, the total channel transmission delay is then calculated as

$$
t_{c d}=\left(c_{c s}+c_{c t} n_{c}\right) / f_{c}
$$

where we have assumed that a connection has already been established between transmitter and receiver and thus ignored bus arbitration and channel setup delays.

\subsection{Driver reception delay model}

We assume that the receiving driver in addition to the parameter $n_{c}$ also receives the parameters $w_{t}$ and $w_{g}$ so

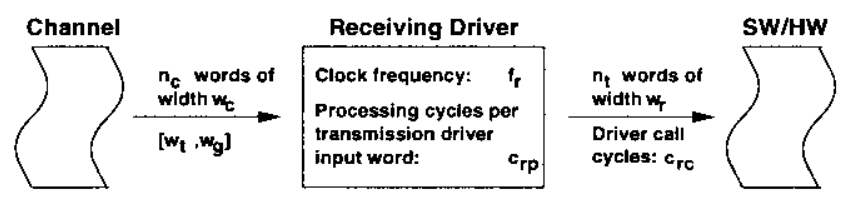

Figure 4. Driver reception parameters.

that it knows how data was packed by the transmitting driver ${ }^{1}$. We will also assume that $w_{r} \geq w_{t}$ and that each unpacked/unsplit word of size $w_{t}$ is put on a single output word of bit width $w_{r}$. Given the clock frequency of the receiving processor $f_{r}$, the number of driver call cycles for reception $c_{r c}$ and the number of reception processing (unpacking/unsplitting/etc.) cycles per transmission driver input word, $c_{r p}$, the formula for driver reception delay simply becomes

$$
t_{r d}=\left(c_{r c}+c_{r p} n_{t}\right) / f_{r}
$$

\subsection{Total transmission delay}

We assume that the driver production of channel words, channel transmission and driver reception of channel words occur in parallel in a pipelined fashion, which means that it is the slowest part that determines the total transmission delay $t_{t}$. We set the maximum delay to

$$
t_{m}=\max \left(t_{t d}, t_{c d}, t_{r d}\right)
$$

and calculate the total transmission delay as

$$
t_{t}=t_{m}+2 \frac{t_{m}}{n_{t}}
$$

where the last term is an approximation of the pipeline startup/completion delay ${ }^{2}$.

\subsection{Burst mode modelling $-n_{c}$ equation}

The preceding sections have assumed that $n_{c}$ and $c_{c s}$ were known. This section and section 2.6 give a detailed derivation of these figures.

In order to be able to handle burst mode transfers, we model $n_{c}$ to consist of $\left(n_{b}-1\right)$ bursts of size $s_{b}$ and a remainder burst of size $s_{r}, 0 \leq s_{r}<s_{b}$ :

$$
n_{c}=\left(n_{b}-1\right) s_{b}+s_{r}
$$

The burst elements all have bit width $w_{c}$. We let the variable $b_{m}$ denote one of three supported burst transfer types, fixed

\footnotetext{
${ }^{1}$ Of course this will not be necessary in the case where the drivers only support fixed values of $w_{t}$ and $w_{g}$ - these values can then be hard coded in the drivers.

${ }^{2}$ As the number of channel words may differ from the number of transmission/reception words, the pipeline startup/completion delay is not modeled accurately by the given term. An exact derivation is outside the scope of this paper - however, it is important to include an estimate of the delay as it may have significance for small transfers.
} 
(each burst has a fixed size), $\max$ (there is a maximum on the burst size, but smaller bursts are allowed) and inf (there is no limit on the burst size). We can now calculate $n_{b}$ and $s_{r}$ as follows: ${ }^{3}$

$$
\begin{aligned}
& n_{b}= \begin{cases}1 & \text { if } b_{m}=\text { inf } \\
\left\lceil n_{c d} / s_{b}\right] & \text { if } b_{m}=\text { fixed, } \max \end{cases} \\
& s_{r}= \begin{cases}s_{b} & \text { if } b_{m}=\text { fixed } \\
n_{c d}-\left(n_{b}-1\right) s_{b} & \text { if } b_{m}=\max , \text { inf }\end{cases}
\end{aligned}
$$

where $n_{c d}$ is the number of actual channel data values corresponding to the $n_{t}$ driver input words of bit width $w_{t}$ which have been packed/split to fit the channel width $w_{c}$. An equation for $n_{c d}$ is derived in section 2.6.

Given the number of synchronization cycles per burst $c_{s b}$ (possibly a fraction) and the number of synchronization cycles per transfer session $c_{s s}$, we can now write the number of channel synchronization cycles $c_{c s}$ as

$$
c_{c s}=\left\lceil n_{b} c_{s b}\right\rceil+c_{s s}
$$

With these definitions, the equations for $n_{c}$ and $c_{c s}$ model the following four variants of burst transfers:

1. Non-burst mode: This is modeled by setting $b_{m}=$ fixed (or max) and the burst size $s_{b}$ to 1 which results in $n_{b}=n_{c d}, s_{r}=1$ and $n_{c}=n_{c d}$. The number of synchronization cycles becomes $c_{c s}=\left\lceil n_{c d} c_{s b}\right\rceil+c_{s s}$ where $c_{s b}$ should now be interpreted as the number of synchronization cycles per channel data word.

2. Burst mode with fixed burst size $s_{b}$. This is modeled by setting $b_{m}=$ fixed which forces the last burst to be of size $s_{b}$ regardless of how many values in that burst are actual data values.

3. Burst mode with maximum burst size $s_{b}$. This is modeled by setting $b_{m}=\max$. The last burst (if any) has size $s_{r}<s_{b}$, but it will still require the same number of burst synchronization cycles as the preceding bursts.

4. Burst mode with unlimited burst size. This is modeled by setting $b_{m}=$ inf and $s_{b}=0$. Then $n_{b}$ becomes 1 indicating a single burst, $s_{r}=n_{c d}$, indicating that $n_{c d}$ data values are to be transferred in the single burst, and $n_{c}=n_{c d}$. The number of synchronization cycles becomes $c_{c s}=\left\lceil c_{s b}\right\rceil+c_{s s}$ so we only spend time on a single set of burst synchronization cycles.

Example 1: (PCI burst mode modelling). Consider a $\mathrm{PCI}$ bus [5] master read transaction of $n_{c d}=1000$ words of width $w_{c}=32$ on a 32 bit wide, $33 \mathrm{Mhz}$ PCI-bus. The PCI-bus supports burst transfers with maximum, fixed as well as unlimited size.

\footnotetext{
${ }^{3}$ In the following, $\lceil x\rceil$ denotes the smallest integer larger than or equal to $\mathrm{x}$ (truncating upwards). $\lfloor x\rfloor$ denotes the largest integer smaller than or equal to $x$ (truncating downwards).
}

We assume a maximum size $\left(b_{m}=\mathbf{m a x}\right)$ burst transfer of size $s_{b}=32$. This ensures a low bus latency that allows other, higher priority, units on the bus to interrupt the transfer. We assume that the bus arbitration latency is 2 clock cycles and that the bus is initially IDLE so that the bus acquisition latency is 0 clock cycles. We set slave device select (DevSel) delay to 1 clock cycle. As the address bus and data bus are multiplexed, the PCI burst transfer consists of an address transfer followed by the (up to) 32 data transfers. For a read transaction, a turnaround cycle is required between the address transfer and the data transfers in order to avoid bus contention. After completion of the burst, an additional IDLE cycle is required. The address transfer and the data transfers each last one clock cycle (assuming zero wait state transfers), except for the first data transfer which lasts 4 clock cycles. We see that the number of synchronization cycles per burst is $c_{s b}=2+0+1$ (DevSel cycle $)+1$ (turnaround cycle $)+$ 3 (extra cycles for first data transfer $)+1$ (IDLE cycle $)=8$. Using (7) and (8), we can now calculate $n_{b}=\left\lceil n_{c d} / s_{b}\right\rceil=$ $\lfloor 1000 / 32\rfloor=32$ and $s_{r}=n_{c d}-\left(n_{b}-1\right) s_{b}=1000-31$. $32=8$. As we set the number of synchronization cycles per session, $c_{s s}$, to zero, we can now use (6) to calculate the number of actually transmitted channel words, $n_{c}$, and (9) to calculate the number of channel synchronization cycles $c_{c s}$ :

$$
\begin{aligned}
& n_{c}=(32-1) \cdot 32+8=1000 \\
& c_{c s}=\lceil 32 \cdot 8\rceil+0=256
\end{aligned}
$$

As the number of transmission cycles per channel word is $c_{c t}=1$, we now use (2) to calculate the channel transmission delay to

$$
t_{c d}=(0+1 \cdot 1000+256+0) /\left(33 \cdot 10^{6}\right)=38 \mu s
$$

\subsection{Data packing/splitting}

In this section we show how the number of channel data words $n_{c d}$ is determined for various packing/splitting schemes.

\subsection{1 $\mathbf{n}_{\mathbf{c d}}$ equation: packing $\left(w_{t} \leq w_{\mathrm{c}}\right)$}

We generalize the process of packing the $n_{t}$ smaller driver input words of width $w_{t}$ into the $n_{c d}$ larger channel data words of width $w_{c}$ to be a two-step process:

1. First split the input words into $n_{1}$ fragments of bit width $w_{g}, w_{g} \leq w_{c}$. If $w_{g} \geq w_{t}$, one input word is put on each fragment as shown in figure 5.C.

2. Then pack as many as possible $\left(n_{2}\right)$ of these fragments onto each channel word. 
The reason for introducing the intermediate first step is that we can then model optimal as well as fast packing with the same equation, as shown below.

Each driver input word occupies $\left\lceil w_{t} / w_{g}\right\rceil$ fragments of width $w_{g}$, so we need to pack a total of $n_{1}=n_{t}\left\lceil w_{t} / w_{g}\right\rceil$ fragments. Each channel word can hold $n_{2}=\left\lfloor w_{c} / w_{g}\right\rfloor$ fragments. The number of required channel words is thus $\left\lceil n_{1} / n_{2}\right\rceil$ which expands to

$$
n_{c d}=\left\lceil\frac{n_{t}\left\lceil w_{t} / w_{g}\right\rceil}{\left\lfloor w_{c} / w_{g}\right\rfloor}\right\rceil, \quad w_{g} \leq w_{c}, w_{t} \leq w_{c}
$$

Figure 5 gives an example of data packing for three different values of $w_{g}$ :

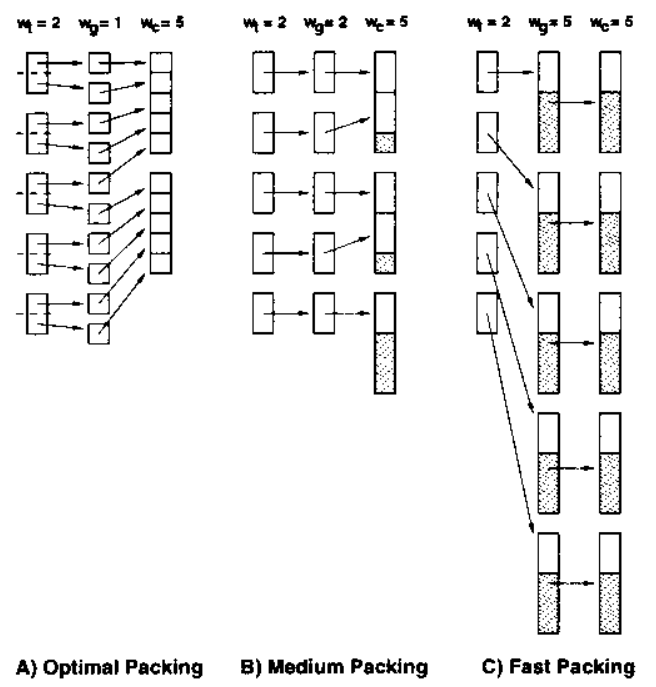

Figure 5. Packing with different granularities.

Optimal packing $\left(w_{g}=1\right)$. Optimal packing is achieved by packing the driver input words in a bit-wise manner. This corresponds to setting the packing granularity $w_{g}$ to 1 . Slack is only possible in the last channel word. (10) reduces to

$$
n_{c d}=\left\lceil\left(n_{t} w_{t}\right) / w_{c}\right\rceil
$$

Medium fast packing $\left(w_{g}=w_{t}\right)$. Medium fast packing is achieved by packing the driver input words in a per input-word manner, i.e. only as many whole input words that can fit in a channel word are put on each channel word. This corresponds to setting the packing granularity $w_{g}$ equal to $w_{t}$. Slack can now occur in each channel word. (10) reduces to

$$
n_{c d}=\left\lceil n_{t} /\left\lfloor w_{c} / w_{t}\right\rfloor\right\rceil
$$

Fast packing $\left(w_{g}=w_{c}\right)$. Fast packing is achieved by packing each input word onto a single channel word. This corresponds to setting the packing granularity $w_{g}$ equal to $w_{c}$. Slack will occur in every channel word if $w_{c}>w_{t}$. Naturally (10) reduces to

$$
n_{c d}=n_{t}
$$

2.6.2 $\mathbf{n}_{\mathrm{cd}}$ equation: splitting $\left(w_{c} \leq w_{t}\right)$

Figure 6 gives an example of data splitting for two different values of $w_{g}$.

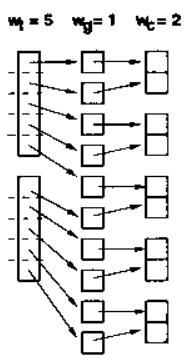

A) Optimal Splitting

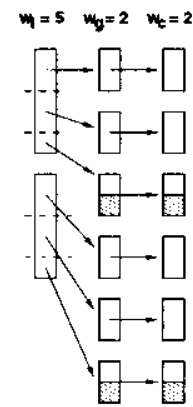

B) Medium Splitting
Figure 6. Splitting with different granularities.

We follow the same two-step approach as in the packing phase and find that equation for $n_{c d}$ becomes identical to (10). This means that (10) covers packing as well as splitting with the only requirement that $w_{g} \leq w_{c}$.

This implies that the equation for optimal splitting $\left(w_{g}=\right.$ 1 ) is identical to (11) and the equation for medium fast splitting $\left(w_{g}=w_{c}\right)$ is identical to (12). There is no "fast splitting" $\left(w_{g}=w_{t}\right)$ case as we cannot in general fit a whole driver data word into the smaller channel words (only when $w_{t}=w_{c}$ ).

\subsubsection{Resulting $\mathbf{n}_{\mathrm{cd}}$ equation}

The final equation for $n_{c d}$ which covers both packing and splitting can now be written as

$$
n_{c d}=\left\lceil\frac{n_{t}\left\lceil w_{t} / w_{g}\right\rceil}{\left\lfloor w_{c} / w_{g}\right\rfloor}\right\rceil, \quad w_{g} \leq w_{c}
$$

This equation models both fast, medium fast and optimal packing/splitting, depending on the parameter $w_{g}$. The packing/splitting time in general depends on $w_{g}$, so the transmission processing delay $c_{t p}$ in (1) and the reception processing delay $c_{r p}$ in (3) are not actually constants but functions of $w_{g}$ :

$$
c_{t p}=F_{t p}\left(w_{g}\right), \quad c_{r p}=F_{r p}\left(w_{g}\right)
$$

The communication model library should provide separate values of $c_{t p}$ and $c_{r p}$ for each supported value of $w_{g}$ or provide the functions $F_{t p}$ and $F_{r p}$ as expressions.

Example 2: (Bit level serial communication modelling). We consider serial RS-232 communication using a serial 
communications controller, for instance a Zilog Z8530 SCC [7] which is configured to perform 8-bit asynchronous communication using 1 stop bit and 1 parity bit. We set the baud rate to 19600 , and assume that we wish to write $n_{t}=1000$ words of bit width $w_{t}=32$. We consider each channel data element to be a single bit, so $w_{c}=w_{g}=1$. (14) gives us the number of channel data words, $n_{c d}$ :

$$
n_{c d}=\lceil(1000\lceil 32 / 1\rceil) /(\lfloor 1 / 1\rfloor)\rceil=32000
$$

We model the channel transfers to consist of bursts of size $s_{b}=8$ and set $b_{m}=$ fixed. There will only be three synchronization cycles per burst (for the implicit start bit and the stop and parity bits) as there is no need to reconfigure the SCC for a write operation each time we transfer a byte and there is no delay between burst (byte) transfers as we can reload the write register while the previous byte is being transferred, so $c_{s b}=3$. Equations (7) and (8) give us $n_{b}=\left\lceil n_{c d} / 8\right\rceil=4000$ and $s_{r}=s_{b}=8$. We assume that the SCC is already properly configured and set $c_{s s}=0$. (6) now gives us $n_{c}=(4000-1) \cdot 8+8=32000$ and (9) gives us $c_{c s}=\lceil 4000 \cdot 3\rceil+0=12000$. Each data element (bit) transfer lasts $c_{t}=1$ clock cycle and the channel clock frequency is $f_{c}=19600$. We can now use (2) to calculate the channel transmission delay to

$$
t_{c d}=(1 \cdot 32000+12000) / 19600=2.2 s
$$

\subsection{Design space exploration}

The preceding examples have focused on demonstrating the modelling capabilities of the communication model. We here give an example of how the model can be used in the design space exploration phase of system level co-synthesis.

Example 3: Consider communication of $\left(n_{t}=1000\right) 16$ bit words $\left(w_{t}=16\right)$ via an $f_{c}=32 \mathrm{Mhz}$ channel of width $w_{c}=32$ using a proprietary protocol with no burst mode ( $b_{m}=$ fixed, $s_{b}=1$ ) and a multiplexed address/data bus with one address transfer per data transfer $\left(c_{s b}=1\right)$ and one clock cycle per transfer $\left(c_{c t}=1\right)$. The receiving processor operates at clock frequency $f_{r}=200 \mathrm{Mhz}$.

First we consider a configuration where we use a fast (and expensive) $f_{t}=100 \mathrm{Mhz}$ transmitting processor that can pack two 16 bit values on each 32 bit channel word using seven processing cycles per transmission word $\left(w_{g}=\right.$ $16, c_{t p}=7$ ). The receiving processor also uses $c_{r p}=7$ cycles per transmission word to unpack the received channel words. All other parameters are set to zero. For this configuration we find that $n_{c d}=500, n_{b}=500, s_{r}=1$, $c_{c s}=500$ and $n_{c}=500$ and can now calculate the transmitting driver delay, channel delay and receiving driver delay to $\left(t_{t d}=70 \mu \mathrm{s}, t_{c d}=31.25 \mu \mathrm{s}, t_{r d}=35 \mu \mathrm{s}\right)$. We see that the transmitting driver is the communication bottleneck $\left(t_{m}=t_{t d}=70 \mu \mathrm{s}\right)$ and find, using (5) the resulting transmission delay to be $\mathbf{t}_{\mathbf{t}}=70 \mu s+2 \cdot(70 \mu s / 1000)=$ $70.14 \mu \mathrm{s}$.

We now consider a configuration where we use a slow (and cheaper) $f_{t}=50 \mathrm{Mhz}$ transmitting processor that only packs one 16 bit value on each 32 bit channel word (i.e. fast packing) thus using only three processing cycles per transmission word $\left(w_{g}=32, c_{t p}=3\right)$. The receiving processor also uses $c_{r p}=3$ unpacking cycles per transmission word. All other parameters are the same as in the previous configuration. We now find that $n_{c d}=1000$, $n_{b}=1000, s_{r}=1, c_{c s}=1000$ and $n_{c}=1000$ which results in $\left(t_{\mathrm{td}}=60 \mu \mathrm{s}, \mathrm{t}_{\mathrm{cd}}=62.5 \mu \mathrm{s}, \mathrm{t}_{\mathrm{rd}}=15 \mu \mathrm{s}\right)$. Here, $t_{m}=62.5 \mu \mathrm{s}$ which results in a total transmission delay of $\mathbf{t}_{\mathbf{t}}=62.5 \mu s+2 \cdot(62.5 \mu s / 1000)=62.6 \mu \mathrm{s}$.

We can conclude that in this case the best choice of transmission processor is the cheap and slow processor, even though it does not utilize the full bus bandwidth and channel transmission time is larger than before. The fact that it spends less time on packing data makes it the better choice. Though being artificial, the example demonstrates that the performance of the drivers have to be balanced with the performance of the channel in order to find the best system configuration.

\section{Conclusion}

We have presented a high level communication estimation model suitable for design space exploration in cosynthesis and have demonstrated its modelling capabilities and intended use. Future work will focus on extending the model to include bus arbitration/acquisition delay in case of buses with multiple drivers and to integrate the communication estimator with partitioning and design space exploration in the LYCOS system.

\section{References}

[1] J.-M. Daveau, T. B. Ismail, and A. A. Jerraya. Synthesis of System-Level Communication by an Allocation-Based Approach. In Eighth International Symposium on System Synthesis, pages $150-155$, September 1995.

[2] J. Madsen, J. Grode, P. V. Knudsen, M. E. Petersen, and A. Haxthausen. LYCOS: the Lyngby Co-Synthesis System. Design Automation for Embedded Systems, 2(2):195 - 235, 1997.

[3] J. Madsen and B. Hald. An Approach to Interface Synthesis. In Proceedings of the Eighth International Symposium on System Synthesis, pages 16-21, 1995.

[4] S. Narayan and D. D. Gajski. Protocol Generation for Communication Channels. In Proceedings of the 3Ith DAC, pages $547-548,1994$.

[5] PCI Special Interest Group. PCI Local Bus Specification, Revision 2.1, June 1995.

[6] J. Zhu, R. Dömer, and D. D. Gajski. Syntax and Semantics of the SpecC Language. In Proceedings of the SASIMI Workshop, pages $75-82,1997$.

[7] Zilog, Inc. SCC/ESCC And ISCC Family Of Products User's Manual, 1997. 